\title{
Active invisibility cloaks in one dimension
}

\author{
Ali Mostafazadeh* \\ Departments of Physics and Mathematics, Koç University, Sartyer 34450, Istanbul, Turkey
}

(Received 19 March 2015; published 12 June 2015)

\begin{abstract}
We outline a general method of constructing finite-range cloaking potentials which render a given finite-range real or complex potential, $v(x)$, unidirectionally reflectionless or invisible at a wave number, $k_{0}$, of our choice. We give explicit analytic expressions for three classes of cloaking potentials which achieve this goal while preserving some or all of the other scattering properties of $v(x)$. The cloaking potentials we construct are the sum of up to three constituent unidirectionally invisible potentials. We discuss their utility in making $v(x)$ bidirectionally invisible at $k_{0}$ and demonstrate the application of our method to obtain antireflection and invisibility cloaks for a Bragg reflector.
\end{abstract}

DOI: 10.1103/PhysRevA.91.063812

PACS number(s): 42.25.Bs, 03.65.Nk, 02.30.Zz

\section{INTRODUCTION}

The possibility of making a scatterer invisible to a distant observer by placing a cloaking device between the two has intrigued both scientists and laymen for a very long time. The discovery of the methods of conformal mapping [1] and transformation optics [2] combined with the mind boggling possibilities offered by metamaterials has recently led to a rapid progress towards the realization of such invisibility cloaks in two and three dimensions [3]. The one-dimensional analog of this problem is typically considered in the context of developing antireflection coatings [4,5]. It involves constructing a finite-range potential $v_{c}(x)$ whose addition to a given finite-range target potential yields a unidirectionally reflectionless or invisible total potential. The purpose of the present article is to give an exact and analytic solution of this problem in such a way that the presence of $v_{c}(x)$ does not alter some or any of the scattering properties of the system in the direction along which it remains visible.

The construction of one-dimensional antireflection cloaks is intimately related to the old problem of characterizing reflectionless potentials. In Ref. [6], Kay and Moses use the powerful tools of inverse scattering theory [7] to provide a systematic method of constructing an infinite class of potentials which are reflectionless at all wave numbers. The best-known member of this class is the reflectionless PöschlTeller potential [8]. The authors of Refs. [9,10] employ the results of Kay and Moses to devise a method of constructing realistic broadband antireflection coatings. Here a serious practical problem is that the reflectionless potentials of Kay and Moses have an infinite range. Therefore, they cannot be realized using material (refractive index profiles) confined to a closed region in space.

Complex absorbing potentials [11,12] offer another means of realizing an antireflection cloak in one dimension. By definition, an absorbing potential has zero (left or right) reflection and transmission coefficients [11]. This implies that it may function as an antireflection cloak from the left (respectively, right), but its presence will drastically alter the scattering properties of the system from the right (respectively, left). In order to provide a more detailed assessment of the

\footnotetext{
*amostafazadeh@ku.edu.tr
}

utility of absorbing potentials in realizing invisibility cloaks and offer a precise formulation of the problem we consider in this article, we briefly survey some of the basic notions of one-dimensional scattering theory.

By definition, the reflection and transmission amplitudes, $R^{l / r}(k)$ and $T(k)$, of a scattering potential $v(x)$ are complex numerical quantities which determine the asymptotic form of the left- and right-incident scattering solutions $\psi_{l / r}(x)$ of the Schrödinger equation

$$
-\psi^{\prime \prime}(x)+v(x) \psi(x)=k^{2} \psi(x),
$$

according to

$$
\begin{aligned}
& \psi_{l}(x)=\left\{\begin{array}{ccc}
e^{i k x}+R^{l}(k) e^{-i k x} & \text { for } & x \rightarrow-\infty, \\
T(k) e^{i k x} & \text { for } & x \rightarrow+\infty,
\end{array}\right. \\
& \psi_{r}(x)=\left\{\begin{array}{ccc}
T(k) e^{-i k x} & \text { for } & x \rightarrow-\infty, \\
e^{-i k x}+R^{r}(k) e^{i k x} & \text { for } & x \rightarrow+\infty .
\end{array}\right.
\end{aligned}
$$

Equations (2) and (3) also apply for the Helmholtz equation, $\psi^{\prime \prime}(x)+k^{2} \mathfrak{n}(x)^{2} \psi(x)=0$, which coincides with Eq. (1) if we relate the refractive index $\mathfrak{n}(x)$ to the potential $v(x)$ via $\mathfrak{n}(x)=\sqrt{1-v(x) / k^{2}}$.

For simplicity of presentation, we choose an arbitrary but fixed value of the wave number $k$, which we label $k_{0}$, and use the symbols $T$ and $R^{l / r}$ to denote $T\left(k_{0}\right)$ and $R^{l / r}\left(k_{0}\right)$, respectively. Note also that we label both the left and the right transmission amplitudes with a $T$, because they coincide [13].

A scattering potential is said to be unidirectionally invisible from the left (respectively, right) if $R^{l}=0 \neq R^{r}$ (respectively, $R^{r}=0 \neq R^{l}$ ) and $T=1$ [14]. We use the term "left-invisible" (respectively, "right-invisible") to refer to such a potential. If a potential is both left- and right-invisible, we call it bidirectionally invisible. Relaxing the condition $T=1$, we similarly define the notions of unidirectional, left-, right-, and bidirectional reflectionlessness.

The study of unidirectionally invisible potentials [14-16] has recently attracted a great deal of attention, because they offer an interesting method of modeling certain one-way optical devices [14]. An equally interesting motivation for exploring these potentials is their unique role in a recently proposed inverse scattering prescription which allows for the construction of finite-range potentials supporting scattering properties of one's choice at any prescribed wave number [17]. 
The following theorem provides a precise statement of this result.

Theorem 1. Let $k_{0}$ be a positive real number, and $\mathscr{R}^{l}$, $\mathscr{R}^{r}$, and $\mathscr{T}$ be arbitrary complex numbers such that $\mathscr{T} \neq 0$. Then there is a finite-range potential, $v(x)$, with the following properties.

(i) The reflection and transmission amplitudes of $v(x)$ at $k=k_{0}$ are, respectively, given by $R^{l / r}=\mathscr{R}^{l, r}$ and $T=\mathscr{T}$.

(ii) If $\mathscr{R}^{l}=\mathscr{R}^{r}=0$ and $\mathscr{T} \neq 1, v(x)$ is the sum of four unidirectionally invisible finite-range potentials with mutually disjoint support [18].

(iii) If $\left|\mathscr{R}^{l}\right|+\left|\mathscr{R}^{r}\right| \neq 0, v(x)$ is the sum of at most three unidirectionally invisible finite-range potentials with mutually disjoint support.

(iv) The support of $v(x)$ can be chosen to be on the left or the right of any point on the real line.

The main technical tool used in the proof of this theorem [17] is the transfer matrix of the one-dimensional potential scattering $[19,20]$. Every solution of the Schrödinger equation (1), for a scattering potential $v(x)$, has the asymptotic form:

$$
\psi(x)=A_{ \pm} e^{i k x}+B_{ \pm} e^{-i k x} \text { as } x \rightarrow \pm \infty,
$$

where $A_{ \pm}$and $B_{ \pm}$are complex coefficients. The transfer matrix of $v(x)$ is, by definition, the $2 \times 2$ matrix $\mathbf{M}$ satisfying

$$
\left[\begin{array}{l}
A_{+} \\
B_{+}
\end{array}\right]=\mathbf{M}\left[\begin{array}{l}
A_{-} \\
B_{-}
\end{array}\right] .
$$

We can express it in terms of the reflection and transmission amplitudes of $v(x)$ according to [20]

$$
\mathbf{M}=\left[\begin{array}{cc}
T-R^{l} R^{r} / T & R^{r} / T \\
-R^{l} / T & 1 / T
\end{array}\right] .
$$

Transfer matrices are extremely useful because of their composition property. Suppose that $v_{ \pm}(x)$ are scattering potentials with support $I_{ \pm}$and transfer matrix $\mathbf{M}_{ \pm}$. If $I_{-}$lies to the left of $I_{+}$(which we denote by $I_{-} \prec I_{+}$), the transfer matrix of $v_{-}(x)+v_{+}(x)$ is given by

$$
\mathbf{M}=\mathbf{M}_{+} \mathbf{M}_{-} .
$$

We can use this relation and Eq. (4) to express the reflection and transmission amplitudes, $R^{r / l}$ and $T$, of $v_{-}(x)+v_{+}(x)$ in terms of those of $v_{ \pm}(x)$. Denoting the latter by $R_{ \pm}^{l / r}$ and $T_{ \pm}$ and using Eqs. (4) and (5), we have

$$
\begin{gathered}
R^{l}=\frac{\left(T_{-}^{2}-R_{-}^{l} R_{-}^{r}\right) R_{+}^{l}+R_{-}^{l}}{1-R_{-}^{r} R_{+}^{l}}, \\
R^{r}=\frac{\left(T_{+}^{2}-R_{+}^{l} R_{+}^{r}\right) R_{-}^{r}+R_{+}^{r}}{1-R_{-}^{r} R_{+}^{l}}, \\
T=\frac{T_{-} T_{+}}{1-R_{-}^{r} R_{+}^{l}} .
\end{gathered}
$$

Suppose that $v(x)$ is a given finite-range potential. If $v_{c}(x)$ is a finite-range potential whose support does not intersect that of $v(x)$ and the addition of $v_{c}(x)$ to $v(x)$ yields a unidirectionally or bidirectionally invisible total potential, $v(x)+v_{c}(x)$, we say that $v_{c}(x)$ is an invisibility cloak for $v(x)$. Similarly, we call $v_{c}(x)$ an antireflection cloak, if $v(x)+v_{c}(x)$ is reflectionless.

For example, consider adding an absorbing potential, $v_{-}(x)$, with $R_{-}^{l}=T_{-}=0$, to a target potential, $v_{+}(x)$, such that $I_{-} \prec$
$I_{+}$. Then according to Eqs. (6) and (8), the left reflection and transmission amplitudes of $v_{-}(x)+v_{+}(x)$ vanish. Therefore, $v_{-}(x)+v_{+}(x)$ is left-reflectionless, but not left-invisible. This argument shows that the absorbing potentials can never be used to construct genuine invisibility cloaks.

Next, consider an antireflection cloaking potential, $v_{c}$, so that the left (or right) reflection amplitude of $v(x)+v_{c}(x)$ vanishes. If the addition of $v_{c}(x)$ to $v(x)$ leaves both the right (respectively, left) reflection amplitude and the transmission amplitude of $v(x)$ intact, we say that $v_{c}(x)$ is an optimal antireflection cloak (or cloaking potential) for $v(x)$. Similarly, we define the notion of an optimal invisibility cloak by demanding that its addition does not alter the right (respectively, left) reflection amplitude of $v(x)$. According to this terminology, the absorbing potentials yield nonoptimal antireflection cloaks. In this article, we give a general method of constructing optimal antireflection and invisibility cloaks for an arbitrary finite-range target potential.

We close this section by summarizing the construction of a concrete model for unidirectionally invisible potentials [17] that we use extensively in the remainder of this article. The importance of such a model is clearly highlighted by Theorem 1 .

Given a nonzero complex number, $\mathcal{R}$, let

$$
\begin{gathered}
v_{\mathcal{R}}^{r}(x):=\left\{\begin{array}{cl}
k^{2} f_{\alpha}\left(x-a_{m}\right) & \text { for } x \in\left[a_{m}, L_{n}+a_{m}\right], \\
0 & \text { for } x \notin\left[a_{m}, L_{n}+a_{m}\right],
\end{array}\right. \\
v_{\mathcal{R}}^{l}(x):=v_{-\mathcal{R}^{*}}^{r}(x)^{*},
\end{gathered}
$$

where

$$
\begin{aligned}
f_{\alpha}(x) & :=\frac{-8 \alpha\left(3-2 e^{2 i k_{0} x}\right)}{e^{4 i k_{0} x}+\alpha\left(1-e^{2 i k_{0} x}\right)^{2}}, \\
a_{m} & :=\frac{(4 m+1) \pi+2 \varphi}{4 k_{0}},
\end{aligned}
$$

$m$ is an arbitrary integer, $n$ and $\alpha$ are, respectively, a positive integer and a positive real number satisfying

$$
|\mathcal{R}|(\alpha+1)^{3}-8 \pi n \alpha=0,
$$

$L_{n}:=\pi n / k_{0}$, and $\varphi$ is the principal argument (phase angle) of $\mathcal{R}$, i.e., $\varphi=-i \ln (\mathcal{R} /|\mathcal{R}|) \in[0,2 \pi)$. Then, $v_{\mathcal{R}}^{l}(x)$ [respectively, $\left.v_{\mathcal{R}}^{r}(x)\right]$ is a left-invisible (respectively, right-invisible) potential with the right (respectively, left) reflection amplitude $\mathcal{R}$ [17]. Note that because $m$ can take arbitrary integer values, we can choose it so that the support of $v_{\mathcal{R}}^{r / l}(x)$ lies to the left or the right of any point or finite interval.

\section{OPTIMAL UNIDIRECTIONAL INVISIBILITY CLOAKS}

Suppose that $v(x)$ is an arbitrary finite-range potential with support $I$ and reflection and transmission amplitudes $R^{l / r}$ and $T$, so that its transfer matrix $\mathbf{M}$ is given by Eq. (4). Theorem 1 implies the existence of finite-range potentials $u_{ \pm}(x)$ with support $I_{ \pm}$and reflection and transmission amplitudes $R_{ \pm}^{l / r}$ and $T_{ \pm}$, such that $I_{-} \prec I \prec I_{+}$and (for $k=k_{0}$ )

$$
\begin{aligned}
& R_{-}^{l}=-\frac{R^{l}}{T^{2}}, \quad R_{-}^{r}=0, \quad T_{-}=\frac{1}{T}, \\
& R_{+}^{l}=0, \quad R_{+}^{r}=-\frac{R^{r}}{T^{2}}, \quad T_{+}=\frac{1}{T} .
\end{aligned}
$$


In view of Eq. (4), the transfer matrix $\mathbf{M}_{ \pm}$of $u_{ \pm}(x)$ at $k_{0}$ takes the form

$$
\mathbf{M}_{-}=\left[\begin{array}{cc}
1 / T & 0 \\
R^{l} / T & T
\end{array}\right], \quad \mathbf{M}_{+}=\left[\begin{array}{cc}
1 / T & -R^{r} / T \\
0 & T
\end{array}\right] .
$$

Because, $I_{-} \prec I \prec I_{+}$, the composition property of the transfer matrix implies that the transfer matrices of $v(x)+u_{-}(x)$ and $v(x)+u_{+}(x)$ are, respectively, given by

$$
\mathbf{M M}_{-}=\left[\begin{array}{cc}
1 & R^{r} \\
0 & 1
\end{array}\right], \quad \mathbf{M}_{+} \mathbf{M}=\left[\begin{array}{cc}
1 & 0 \\
-R^{l} & 1
\end{array}\right] .
$$

This shows that $v(x)+u_{-}(x)$ is left-invisible and has the same right reflection amplitude as $v(x)$, and $v(x)+u_{+}(x)$ is rightinvisible and has the same left reflection amplitude as $v(x)$. Therefore, $u_{ \pm}(x)$ are unidirectionally reflectionless potentials that serve as optimal invisibility cloaks for $v(x)$.

Suppose that not both $R^{l}$ and $R^{r}$ vanish. Then, according to Theorem 1 , we can construct $u_{ \pm}(x)$ using three unidirectionally invisible potentials, which we denote by $u_{j \pm}(x)$ with $j=1,2,3$.

We identify $u_{j \pm}(x)$ with a finite-range potential having the domain $I_{j \pm}$ and the transfer matrix $\mathbf{M}_{j \pm}$ such that $I_{1 \pm} \prec I_{2 \pm} \prec$ $I_{3 \pm}$ and

$$
\begin{aligned}
& \mathbf{M}_{1-}=\left[\begin{array}{cc}
1 & \frac{T(T-1)}{R^{l}} \\
0 & 1
\end{array}\right], \quad \mathbf{M}_{2-}=\left[\begin{array}{cc}
1 & 0 \\
\frac{R^{l}}{T} & 1
\end{array}\right], \\
& \mathbf{M}_{3-}=\left[\begin{array}{cc}
1 & \frac{1-T}{R^{l}} \\
0 & 1
\end{array}\right], \quad \mathbf{M}_{1+}=\left[\begin{array}{cc}
1 & 0 \\
\frac{T-1}{R^{r}} & 1
\end{array}\right], \\
& \mathbf{M}_{2+}=\left[\begin{array}{cc}
1 & -\frac{R^{r}}{T} \\
0 & 1
\end{array}\right], \quad \mathbf{M}_{3+}=\left[\begin{array}{cc}
1 & 0 \\
\frac{T(1-T)}{R^{r}} & 1
\end{array}\right] .
\end{aligned}
$$

Then, it is easy to check that $\mathbf{M}_{ \pm 3} \mathbf{M}_{ \pm 2} \mathbf{M}_{ \pm 1}=\mathbf{M}_{ \pm}$. This shows that we can take

$$
u_{ \pm}(x)=u_{1 \pm}(x)+u_{2 \pm}(x)+u_{3 \pm}(x) .
$$

Notice that the construction of the optimal cloaking potential $u_{-}(x)$ [respectively, $u_{+}(x)$ ] is desirable only if $R^{l} \neq 0$ (respectively, $R^{r} \neq 0$ ). According to Eqs. (18)-(20), $\mathbf{M}_{j-}$ (respectively, $\mathbf{M}_{j+}$ ) exists precisely for this case.

The above analysis reduces the construction of the optimal invisibility clocking potentials $u_{ \pm}(x)$ to that of the finite-range unidirectionally invisible potentials $u_{j \pm}(x)$. We can employ the model introduced in Eqs. (9) and (10) to give explicit expressions for the latter. The potentials $u_{ \pm}(x)$ that we obtain in this way define optimal invisibility cloaks that eliminate the reflection of the plane waves with wave number $k_{0}$ from one direction, set the transmission amplitude to unity, but do not affect the reflection amplitude of $v(x)$ from the other direction. These invisibility cloaks correspond to planar slabs consisting of three optically active layers with a particular locally periodic gain-loss profile and adjustable gaps in between.

\section{NONOPTIMAL UNIDIRECTIONAL INVISIBILITY CLOAKS}

In this section we examine cloaking potentials that render the original potential invisible from one direction but do alter its reflection feature from the other direction.
Consider the finite range potentials $\check{u}_{ \pm \ell}(x)$ with $\ell=1,2$ such that their support $\breve{I}_{ \pm \ell}$ and transfer matrix $\check{\mathbf{M}}_{ \pm \ell}$ satisfy

$$
\begin{gathered}
\check{I}_{1-} \prec \check{I}_{2-} \prec I \prec \check{I}_{1+} \prec \check{I}_{2+}, \\
\check{\mathbf{M}}_{1-}=\left[\begin{array}{cc}
1 & 0 \\
\frac{R^{l}}{T} & 1
\end{array}\right], \quad \check{\mathbf{M}}_{2-}=\left[\begin{array}{cc}
1 & \frac{1-T}{R^{l}} \\
0 & 1
\end{array}\right], \\
\check{\mathbf{M}}_{1+}=\left[\begin{array}{cc}
1 & 0 \\
\frac{T-1}{R^{r}} & 1
\end{array}\right], \quad \check{\mathbf{M}}_{2+}=\left[\begin{array}{cc}
1 & -\frac{R^{r}}{T} \\
0 & 1
\end{array}\right] .
\end{gathered}
$$

Comparing these relations with Eq. (4), we see that $\check{u}_{ \pm \ell}(x)$ are unidirectionally invisible potentials with the reflection amplitude $\check{R}_{j \pm}^{l / r}$ given by $\check{R}_{1 \pm}^{r}=\check{R}_{2 \pm}^{l}=0$ and

$$
\begin{aligned}
& \check{R}_{1-}^{l}=-\frac{R^{l}}{T}, \quad \check{R}_{2-}^{r}=\frac{1-T}{R^{l}}, \\
& \check{R}_{1+}^{l}=\frac{1-T}{R^{r}}, \quad \check{R}_{2+}^{r}=-\frac{R^{r}}{T} .
\end{aligned}
$$

Now, let $\check{u}_{ \pm}(x):=\check{u}_{1 \pm}(x)+\check{u}_{2 \pm}(x)$. Then, in view of Eqs. (22)-(24), the transfer matrix of the potentials $v(x)+$ $\check{u}_{-}(x)$ and $v(x)+\check{u}_{+}(x)$ are, respectively, given by

$$
\begin{gathered}
\mathbf{M} \check{\mathbf{M}}_{2-} \check{\mathbf{M}}_{1-}=\left[\begin{array}{ccc}
1 & R^{r}-\frac{T(T-1)}{R^{l}} \\
0 & 1
\end{array}\right], \\
\check{\mathbf{M}}_{2+} \check{\mathbf{M}}_{1+} \mathbf{M}=\left[\begin{array}{cc}
1 & 0 \\
-R^{l}+\frac{T(T-1)}{R^{r}} & 1
\end{array}\right] .
\end{gathered}
$$

These in turn identify $\check{u}_{ \pm}(x)$ with a pair of invisibility cloaks which are not necessarily optimal; the addition of $\check{u}_{-}(x)$ makes $v(x)$ left-invisible but changes its right reflection amplitude to $R^{r}-T(T-1) / R^{l}$, while the addition of $\check{u}_{+}(x)$ makes $v(x)$ right-invisible and changes its left reflection amplitude to $R^{l}-$ $T(T-1) / R^{r}$.

Again we can use the model described by Eqs. (9) and (10) to give explicit formulas for the constituent unidirectionally invisible potentials $\check{u}_{\ell \pm}(x)$ and consequently the cloaking potentials $\check{u}_{ \pm}(x)$. The advantage of the latter over their optimal analogs, namely $u_{ \pm}(x)$, is that they correspond to planar slabs consisting of two optically active layers.

\section{OPTIMAL UNIDIRECTIONAL ANTIREFLECTION CLOAKS AND BIDIRECTIONAL INVISIBILITY}

It is not difficult to see that we can construct (single-layer) cloaking slabs described by unidirectionally invisible potentials $w_{ \pm}(x)$ whose addition to $v(x)$ yields a unidirectionally reflectionless potential. As suggested by Eq. (17), we can identify $w_{ \pm}(x)$ with the finite-range potentials whose support $J_{ \pm}$and transfer matrix $\mathbf{N}_{ \pm}$fulfill $J_{-} \prec I \prec J_{+}$and

$$
\mathbf{N}_{-}=\left[\begin{array}{cc}
1 & 0 \\
R^{l} & 1
\end{array}\right], \quad \mathbf{N}_{+}=\left[\begin{array}{cc}
1 & -R^{r} \\
0 & 1
\end{array}\right],
$$

i.e., $w_{-}(x)$ is a right-invisible potential with left reflection amplitude $-R^{l}$, while $w_{+}(x)$ is a left-invisible potential with right reflection amplitude $-R^{r}$.

Using Eqs. (4) and (29) to compute the transfer matrix of $v(x)+w_{ \pm}(x)$, we find that the $v(x)+w_{-}(x)$ [respectively, $\left.v(x)+w_{+}(x)\right]$ is a left (respectively, right) reflectionless 
potential with the same right (respectively, left) reflection and transmission amplitudes as $v(x)$. Therefore, $w_{ \pm}(x)$ are optimal reflectionless cloaking potentials. A concrete choice for $w_{-}(x)$ [respectively, $w_{+}(x)$ ] is the potential $v_{-R^{l}}^{r}(x)$ of Eq. (9) [respectively, $v_{-R^{r}}^{l}(x)$ of Eq. (10)].

Next, consider using the cloaking potentials $\hat{u}_{ \pm}(x)$ together with $w_{\mp}(x)$. If we adjust the parameters of the latter so that the left (respectively, right) reflection amplitude of $w_{-}(x)$ [respectively, $w_{+}(x)$ ] coincides with $-R^{r}+T(T-1) / R^{l}$ [respectively, $-R^{l}+T(T-1) / R^{r}$ ], both the potentials $v(x)+$ $\hat{u}_{ \pm}(x)+w_{\mp}(x)$ become bidirectionally invisible, i.e., their transfer matrix coincides with the identity matrix, at $k=k_{0}$. Notice that the support of $v(x)$ lies between those of $\hat{u}_{ \pm}(x)$ and $w_{\mp}(x)$; more specifically, we have $\breve{I}_{1-} \prec \check{I}_{2-} \prec I \prec J_{+}$ and $J_{-} \prec I \prec \check{I}_{1+} \prec \check{I}_{2+}$.

\section{APPLICATION TO A BRAGG REFLECTOR}

Consider the application of the results of the preceding sections in the construction of invisibility and antireflection cloaks for the following locally periodic potential:

$$
v_{b}(x):=\left\{\begin{array}{cc}
k^{2}\left\{1-\left[\mathfrak{n}_{0}+\mathfrak{n}_{1} \sin (K x)\right]^{2}\right\} & \text { for } x \in[0, L], \\
0 & \text { for } x \notin[0, L],
\end{array}\right.
$$

where $\mathfrak{n}_{0}=2.29, \mathfrak{n}_{1}=0.01, K=2 \pi / \Lambda, \Lambda=232 \mathrm{~nm}$, and $L=250 \Lambda=58 \mu \mathrm{m}$. This corresponds to a $1.06 \mu \mathrm{m}$ Bragg reflector made out of $\mathrm{ZnS}$ [4]. Our aim is to construct various cloaking potentials to achieve unidirectional reflectionlessness and invisibility of the cloaked system at the wavelength $\lambda_{0}=$ $1064 \mathrm{~nm}$. Because of the symmetry of the problem, we confine our attention to the construction of left-invisibility and leftantireflection cloaking potentials.

We can easily use the results of Refs. [21,22] to compute the reflection and transmission amplitudes of the potential (30) numerically. For $\lambda_{0}=1064 \mathrm{~nm}$, which corresponds to $k_{0}=$ $5.90525 / \mu \mathrm{m}$, they are given by

$$
\begin{aligned}
& R_{b}^{l}=-0.9102811+0.2133993 i, \\
& R_{b}^{r}=-0.4876307+0.7977251 i, \\
& T_{b}=-0.2402088+0.2610598 i .
\end{aligned}
$$

In particular, for the reflection and transmission coefficients, $\left|R_{b}^{l / r}\right|^{2}$ and $\left|T_{b}\right|^{2}$, we find

$$
\left|R_{b}^{l / r}\right|^{2}=1-\left|T_{b}\right|^{2} \approx 87 \% \text {. }
$$

\section{A. Antireflection cloaking potential $w_{-}(x)$}

As we mentioned above, a concrete realization of $w_{-}(x)$ is provided by the potentials of the form (9) with $\mathcal{R}=-R_{b}^{l}$, i.e.,

$$
w_{-}(x)=v_{-R_{b}^{l}}^{r}(x) .
$$

We only need to make proper choices for the parameters $m, n$, and $\alpha$ appearing in the expression for $v_{-R_{b}^{l}}^{r}(x)$.

Figure 1 shows plots of the left reflection coefficient for the Bragg reflector potential (30) and the cloaking potential (35) for $m=-19048, n=250$, and $\alpha=1.488700 \times 10^{-4}$. The latter corresponds to a $133-\mu \mathrm{m}$-thick slab placed at a distance

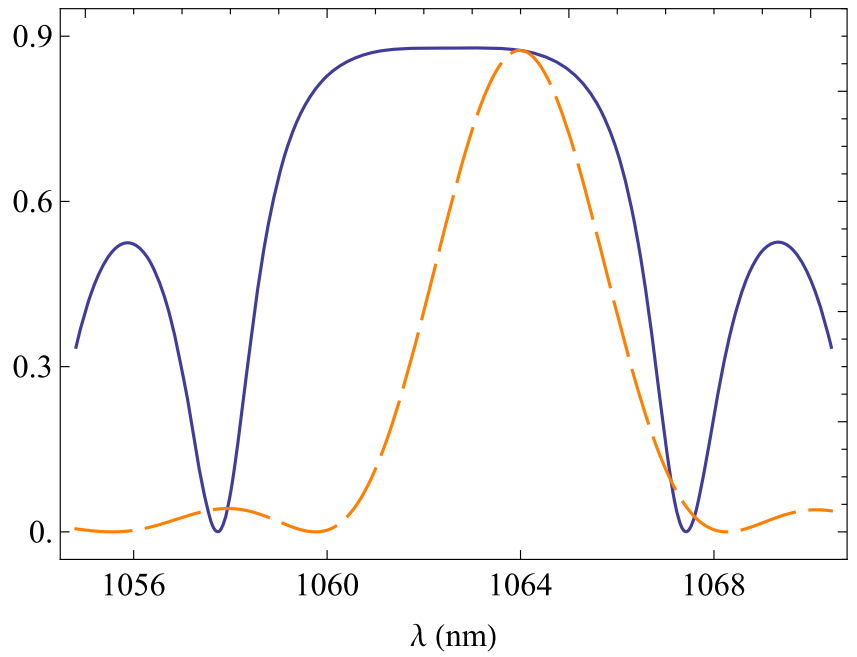

FIG. 1. (Color online) Graphs of the left reflection coefficient $\left|R^{l}\right|^{2}$ for the Bragg reflector potential $v_{b}(x)$ (navy solid curve) and the antireflection cloaking potential $w_{-}(x)$ (orange dashed curve) of Sec. V A.

of about $1 \mathrm{~cm}$ to the left of the Bragg reflector. Note that by taking smaller and larger values of $m$ we can adjust the distance between the Bragg reflector and the clocking slab. A remarkable property of the cloaking potential (35) is that it displays broadband right-invisibility; in the spectral range depicted in Fig. 1, $\left|R^{r}\right|$ and $|T-1|$ are, respectively, bounded by $9 \times 10^{-7}$ and $6 \times 10^{-6}$.

Figure 2 shows the graphs of the left reflection coefficient for the Bragg reflector potential $v_{b}(x)$, the clocking potential $w_{-}(x)$, and $v_{b}(x)+w_{-}(x)$ for the above-given choice of the parameters $n, m$, and $\alpha$. The cloaking effect happens to be restricted to a narrow spectral band of less than $1 \mathrm{~nm}$ in width. Notice however that this is an extreme example where we attempt to make a mirror reflectionless. For the wavelengths outside the range [1054 nm, $1074 \mathrm{~nm}$ ], the cloaking potential

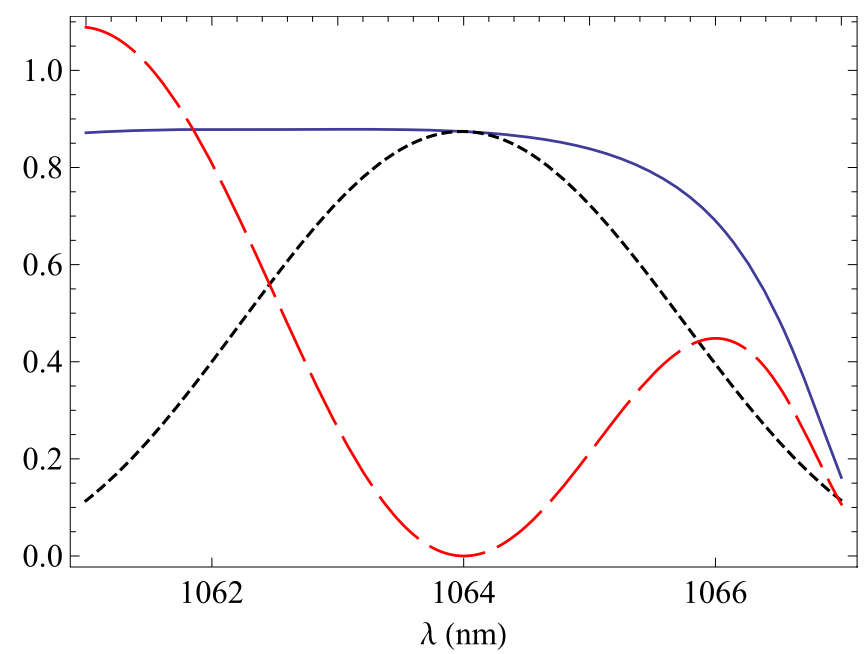

FIG. 2. (Color online) Graphs of the left reflection coefficient $\left|R^{l}\right|^{2}$ for the Bragg reflector potential $v_{b}(x)$ (navy solid curve), the antireflection cloaking potential $w_{-}(x)$ (black dotted curve), and the cloaked potential $v_{b}(x)+w_{-}(x)$ (red dashed curve) of Sec. V A. 


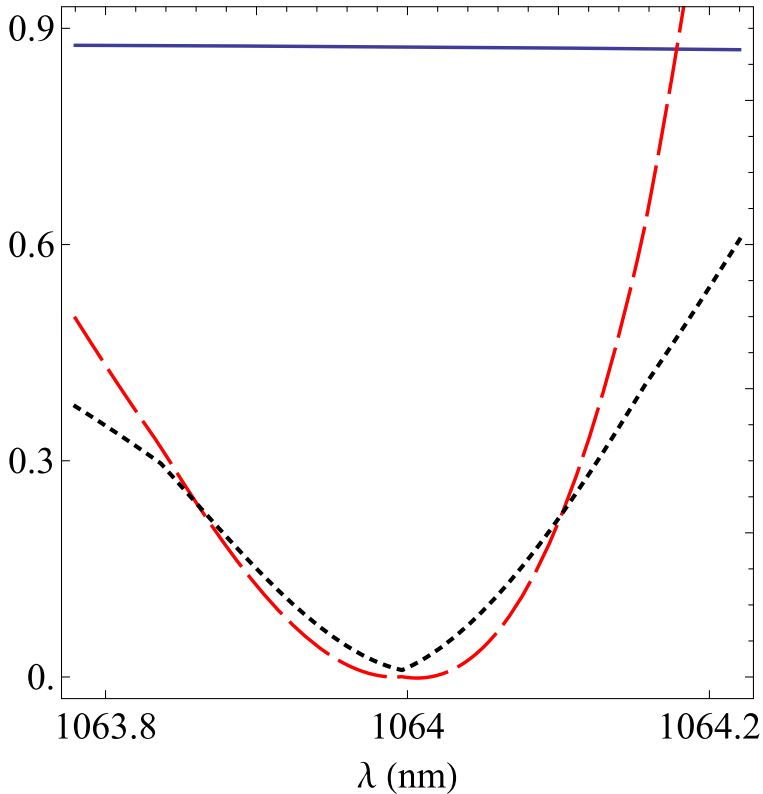

FIG. 3. (Color online) Graph of $\left|R^{l}\right|^{2}$ for the Bragg reflector potential $v_{b}(x)$ (navy solid curve), and graphs of $\left|R^{l}\right|^{2}$ (red dashed curve) and $|T-1|$ (black dotted curve) for the potential $v_{b}(x)+$ $\check{u}_{-}(x)$ of Sec. V B.

is essentially bidirectionally invisible and the reflection and transmission coefficients of $v_{b}(x)+w_{-}(x)$ coincide with those of $v_{b}(x)$. Within this range, their left reflection coefficients differ appreciably, but their right reflection coefficients and the transmission coefficients agree to an extremely high degree of accuracy (their difference is less than $10^{-5}$ ).

\section{B. Nonoptimal invisibility cloaking potential $\check{u}_{-}(x)$}

In order to construct the cloaking potential $\check{u}_{-}(x)$ for the Bragg reflector potential $v_{b}(x)$, we use the model given by Eqs. (9) and (10) to determine a concrete realization of the unidirectional invisible potentials $\check{u}_{1-}(x)$ and $\check{u}_{2-}(x)$. In view of Eq. (25), we set

$$
\check{u}_{1-}(x)=v_{\mathcal{R}_{1}}^{r}(x), \quad \check{u}_{2-}(x)=v_{\mathcal{R}_{2}}^{l}(x)=v_{-\mathcal{R}_{2}^{*}}^{r}(x)^{*},
$$

where

$$
\begin{aligned}
& \mathcal{R}_{1}:=-R_{b}^{l} / T_{b}=-2.180072-1.480919 i \\
& \mathcal{R}_{2}:=\left(1-T_{b}\right) / R_{b}^{l}=-1.355199-0.030912 i .
\end{aligned}
$$

We also take the following values for the parameters $m, n$, and $\alpha$, which enter the expression for $v_{\mathcal{R}_{1}}^{r}(x)$ and $v_{-\mathcal{R}_{2}^{*}}^{r}(x)^{*}$, respectively:

$$
\begin{array}{lll}
m_{1}=-38094, & n_{1}=250 & \alpha_{1}=4.199808 \times 10^{-4} \\
m_{2}=-19048, & n_{2}=250 & \alpha_{2}=1.158825 \times 10^{-4} .
\end{array}
$$

These correspond to a pair of $133-\mu \mathrm{m}$-thick parallel planar slabs placed on the left of the original Bragg reflector slab such that the distance between neighboring slabs is $1 \mathrm{~cm}$.

Figure 3 shows the graphs of the left reflection coefficient for $v_{b}(x)$ before and after adding the cloaking potential,

$$
\check{u}_{-}(x)=\check{u}_{1-}(x)+\check{u}_{2-}(x)=v_{\mathcal{R}_{1}}^{r}(x)+v_{-\mathcal{R}_{2}^{*}}^{r}(x)^{*},
$$

and the graph of $|T-1|$ for $v_{b}(x)+\check{u}_{-}(x)$. These graphs confirm the left-invisibility of $v_{b}(x)+\check{u}_{-}(x)$ within a very narrow spectral band centered at $\lambda=1064 \mathrm{~nm}$.

\section{Optimal invisibility cloaking potential $u_{-}(x)$}

To construct the cloaking potential $u_{-}(x)$ for $v_{b}(x)$, we need to identify the potentials $u_{j-}(x)$ by fixing the values of the parameters $\mathcal{R}, n, \alpha$, and $m$ in Eqs. (9) and (10) appropriately. Let us label the values of these parameters for $u_{j-}(x)$, respectively, by $\mathcal{R}_{j}, n_{j}, \alpha_{j}$, and $m_{j}$, so that

$$
\begin{aligned}
& u_{1-}(x)=v_{\mathcal{R}_{1}}^{l}(x)=v_{-\mathcal{R}_{1}^{*}}^{r}(x)^{*}, \\
& u_{2-}(x)=v_{\mathcal{R}_{2}}^{r}(x), \\
& u_{3-}(x)=v_{\mathcal{R}_{3}}^{l}(x)=v_{-\mathcal{R}_{3}^{*}}^{r}(x)^{*} .
\end{aligned}
$$

Then according to Eqs. (18), (19), (31), and (33),

$$
\begin{aligned}
& \mathcal{R}_{1}=T_{b}\left(T_{b}-1\right) / R_{b}^{l}=-0.333601+0.346363 i, \\
& \mathcal{R}_{2}=-R_{b}^{l} / T_{b}=-2.180072-1.480919 i, \\
& \mathcal{R}_{3}=\left(1-T_{b}\right) / R_{b}^{l}=-1.355199-0.030912 i .
\end{aligned}
$$

If we take $n_{1}=n_{2}=n_{3}=250$, the potentials $u_{j-}(x)$ correspond to $133 \mu \mathrm{m}$-thick active slabs, and we find

$$
\begin{array}{ll}
\alpha_{1}=7.655383 \times 10^{-5}, & m_{1}=-57142, \\
\alpha_{2}=4.199808 \times 10^{-4}, & m_{2}=-38094, \\
\alpha_{3}=2.158825 \times 10^{-4}, & m_{3}=-19048,
\end{array}
$$

where we have made use of Eqs. (13) and (12) and chosen $m_{j}$ in such a way that the distance between neighboring slabs associated with the potentials $u_{j \pm}(x)$ and $v_{b}(x)$ is $1 \mathrm{~cm}$.

Figure 4 provides a graphical demonstration of the effect of adding the cloaking potential $u_{-}(x)$ to the Bragg reflector

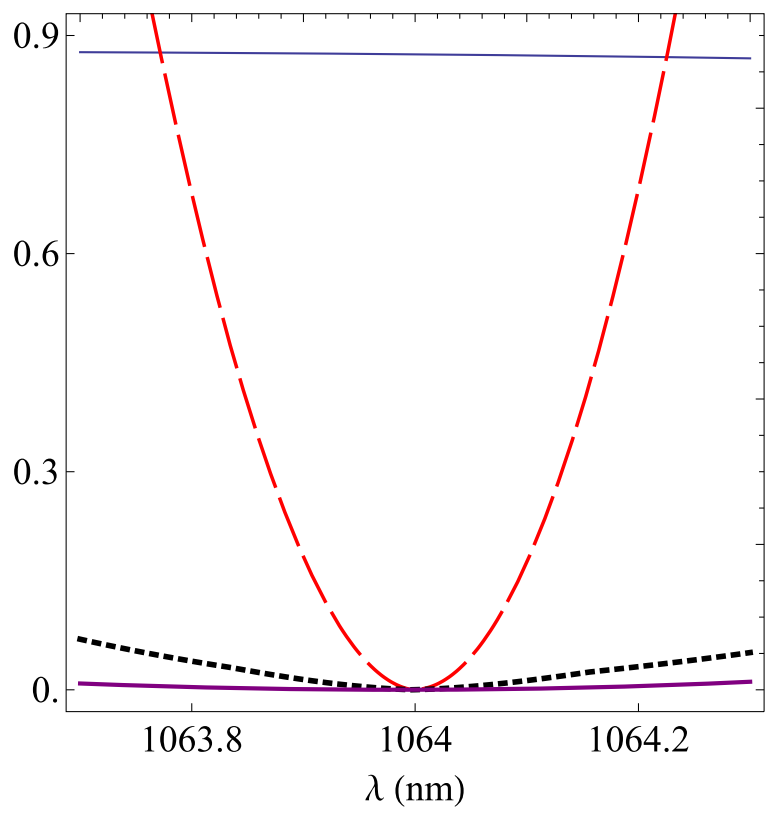

FIG. 4. (Color online) Graph of $\left|R^{l}\right|^{2}$ for the Bragg reflector potential $v_{b}(x)$ of Eq. (30) (thin, navy solid curve), graphs of $\left|R^{l}\right|^{2}$ (red dashed curve) and $|T-1|$ (black dotted curve) for the cloaked potential $v_{b}(x)+u_{-}(x)$ of Sec. V C, and the graph of $\left|R^{r}-R_{b}^{r}\right|$ (thick purple curve), where $R^{r}$ and $R_{b}^{r}$ are, respectively, the right reflection amplitude of $v_{b}(x)+u_{-}(x)$ and $v_{b}(x)$. 
potential $v_{b}(x)$. It confirms that indeed $v_{b}(x)+u_{-}(x)$ is left-invisible and has the same right reflection amplitude as $v_{b}(x)$. Our numerical calculations show that, for $\lambda=1064 \mathrm{~nm}$, the reflection and transmission amplitudes of $v_{b}(x)+u_{-}(x)$ satisfy $\left|R^{l}\right|^{2}<4.1 \times 10^{-4},|T-1|<5.0 \times 10^{-5}$, and $\mid R^{r}-$ $R_{b}^{r} \mid<2.6 \times 10^{-5}$.

\section{CONCLUDING REMARKS}

The discovery that certain complex scattering potentials can display unidirectional invisibility is important, for they possess nonreciprocal scattering features which might find important applications in optical circuitry. This constituted the initial motivation for the study of these potentials $[14,16]$. Recently, it became clear that unidirectional invisible potentials played a central role in scattering theory, in the sense that they could be used to construct scattering potentials with any desired scattering properties at any prescribed wave number [17].

In the present article we have employed this property of unidirectional invisible potentials to outline a method for the construction of various cloaking potentials which make a given finite-range real or complex potential unidirectionally reflectionless or invisible at an arbitrary wave number of our choice. In particular, we obtained optimal antireflection and invisibility cloaks which cannot be constructed using absorbing potentials. Our method relies on the use of up to three finite-range unidirectionally invisible potentials for which we give explicit close-form expressions.

In the application of our method for the Bragg reflector, the cloaking action is confined to a spectral band centered at the desired wavelength which can be as narrow as a small fraction of a nanometer. However, we would like to stress that the width of this band can be increased by a proper choice of the constituent unidirectionally invisible potentials. In this article, we have confined our attention to the class of unidirectionally invisible potentials (9) and made essentially arbitrary choices for their parameters. Our method should, in principle, allow for broadband cloaking action, if we can supplement it with an optimization procedure to select the optimal choices for the constituent unidirectionally invisible potentials. This is a subject of a future investigation.

Finally, we wish to point out that the optimal antireflection and invisibility cloaks should offer useful applications in optical modulation and even display technologies, because they can be used as wavelength-sensitive one-way control devices.

\section{ACKNOWLEDGMENTS}

I would like to thank Aref Mostafazadeh for fruitful discussions. This work has been supported by the Scientific and Technological Research Council of Turkey (TÜBITAK), within the framework of Project No. 112T951, and by the Turkish Academy of Sciences (TÜBA).
[1] U. Leonhardt, Science 132, 1777 (2006).

[2] J. B. Pendry, D. Schurig, and D. R. Smith, Science 312, 1780 (2006).

[3] D. Schuring, J. J. Mock, B. J. Justice, S. A. Cummer, J. B. Pendry, A. F. Starr, and D. R. Smith, Science 314, 977 (2006); M. Rahm, S. A. Cummer, D. Schurig, J. B. Pendry, and D. R. Smith, Phys. Rev. Lett. 100, 063903 (2008); J. Valentine, J. Li, T. Zentgraf, G. Bartal, and X. Zhang, Nat. Mater. 8, 568 (2009); S. Xu, X. Cheng, S. Xi, R. Zhang, H. O. Moser, Z. Shen, Y. Xu, Z. Huang, X. Zhang, F. Yu, B. Zhang, and H. Chen, Phys. Rev. Lett. 109, 223903 (2012); N. Wang, M. Mukhtar, Y. Ma, R. Huang, and C. K. Ong, Europhys. Lett. 104, 14003 (2013).

[4] P. Yeh, Optical Waves in Layered Media (Wiley, Hoboken, NJ, 2005).

[5] W. H. Southwell, Opt. Lett. 11, 584 (1983); P. V. Adamson, Tech. Phys. Lett. 26, 1003 (2000); D. Poitras and J. A. Dobrowolski, Appl. Opt. 43, 1286 (2004).

[6] I. Kay and H. E. Moses, J. Appl. Phys. 27, 1503 (1956).

[7] K. Chadan and P. C. Sabatier, Inverse Problems in Quantum Scattering Theory (Springer, New York, 1989).

[8] S. Flügge, Practial Quantum Mechanics (Springer-Verlag, Berlin, 1971), Vol. 1.

[9] S. Dutta Gupta and G. S. Agarwal, Opt. Express 15, 9614 (2007).

[10] L. V. Thekkekara, V. G. Achanta, and S. Dutta Gupta, Opt. Express 22, 17382 (2014).

[11] J. G. Muga, J. P. Palao, B. Navarro, and I. L. Egusquiza, Phys. Rep. 395, 357 (2004).
[12] U. V. Riss and H.-D. Meyer, J. Phys. B 26, 4503 (1993); N. Moiseyev, ibid. 31, 1431 (1998); O. Shemer, D. Brisker, and N. Moiseyev, Phys. Rev. A 71, 032716 (2005).

[13] A. Mostafazadeh and H. Mehri-Dehnavi, J. Phys. A 42, 125303 (2009).

[14] Z. Lin, H. Ramezani, T. Eichelkraut, T. Kottos, H. Cao, and D. N. Christodoulides, Phys. Rev. Lett. 106, 213901 (2011).

[15] L. Poladian, Phys. Rev. E 54, 2963 (1996); M. Greenberg and M. Orenstein, Opt. Lett. 29, 451 (2004); M. Kulishov, J. M. Laniel, N. Belanger, J. Azana, and D. V. Plant, Opt. Exp. 13, 3068 (2005).

[16] S. Longhi, J. Phys. A 44, 485302 (2011); H. F. Jones, ibid. 45, 135306 (2012); R. Uzdin and N. Moiseyev, Phys. Rev. A 85, 031804 (2012); A. Regensburger, C. Bersch, M. A. Miri, G. Onishchukov, D. N. Christodoulides, and U. Peschel, Nature (London) 488, 167 (2012); A. Mostafazadeh, Phys. Rev. A 87, 012103 (2013); 89, 012709 (2014).

[17] A. Mostafazadeh, Phys. Rev. A 90, 023833 (2014); 90, 055803 (2014).

[18] The support of the function $v(x)$ of the real variable $x$ is the smallest closed interval outside of which $v(x)$ vanishes.

[19] L. L. Sánchez-Soto, J. J. Monzóna, A. G. Barriuso, and J. F. Cariñena, Phys. Rep. 513, 191 (2012).

[20] A. Mostafazadeh, Phys. Rev. Lett. 102, 220402 (2009).

[21] A. Mostafazadeh, Ann. Phys. (NY) 341, 77 (2014).

[22] P. Yeh, A. Yariv, and C.-S. Hong, J. Opt. Soc. Am. 67, 423 (1977); P. Pereyra, Phys. Rev. Lett. 80, 2677 (1998); D. J. Griffiths and C. A. Steinke, Am. J. Phys. 69, 137 (2001). 